\title{
Validation of 3D Code KATRIN For Fast Neutron Fluence Calculation of VVER-1000 Reactor Pressure Vessel by Ex-Vessel Measurements and Surveillance Specimens Results
}

\author{
A. Dzhalandinov ${ }^{1}$, V. Tsofin ${ }^{1}$, V. Kochkin ${ }^{2}$, P. Panferov ${ }^{2}$, A. Timofeev ${ }^{2}$, A. Reshetnikov $^{2}$, \\ D. Makhotin' ${ }^{2}$ D. Erak ${ }^{2}$, and A. Voloschenko ${ }^{3}$ \\ ${ }^{1}$ OKB “GIDROPRESS”, 142103, Ordzhonikidze street, 21, Podolsk, Russia \\ ${ }^{2}$ National Research Centre « Kurchatov Institute », 123182, Kurchatov Sq., 1, Moscow, Russia \\ ${ }^{3}$ Keldysh Institute of Applied Mathematics, 125047, Miusskaya Sq., 4, Moscow, Russia
}

\begin{abstract}
Usually the synthesis of two-dimensional and one-dimensional discrete ordinate calculations is used to evaluate neutron fluence on VVER-1000 reactor pressure vessel (RPV) for prognosis of radiation embrittlement. But there are some cases when this approach is not applicable. For example the latest projects of VVER-1000 have upgraded surveillance program. Containers with surveillance specimens are located on the inner surface of RPV with fast neutron flux maximum. Therefore, the synthesis approach is not suitable enough for calculation of local disturbance of neutron field in RPV inner surface behind the surveillance specimens because of their complicated and heterogeneous structure. In some cases the VVER-1000 core loading consists of fuel assemblies with different fuel height and the applicability of synthesis approach is also ambiguous for these fuel cycles. Also, the synthesis approach is not enough correct for the neutron fluence estimation at the RPV area above core top. Because of these reasons only the 3D neutron transport codes seem to be satisfactory for calculation of neutron fluence on the VVER-1000 RPV. The direct 3D calculations are also recommended by modern regulations.
\end{abstract}

\section{Description of 3D Code KATRIN}

In this paper the 3D discrete ordinate code KATRIN [1,2] is tested for calculation of fast neutron fluence on VVER-1000 Reactor Pressure Vessel (RPV) by discrete ordinate method.

The code KATRIN solves the multigroup transport equation for neutrons, photons and charged particles in $3 \mathrm{D} x, y, z$ and $r, \theta, z$ geometries. The transport equation for charged particles can be solved in the continuous slowing-down approximation. The scattering anisotropy can be treated in the $\mathrm{P}_{\mathrm{L}}$ approximation. The adjoint solution of the problem can be also obtained (for neutral particles only). The principal application is the solving the deep-penetration transport problems, typical for radiation protection and shielding calculations. The fission problems (subcritical boundary value problem and $k_{\text {eff }}$ problem), problems with upscattering (the thermalization problem, etc.) and hadron cascade problems 
can be also solved. The possibility to calculate spectra/doses in a void outside KATRIN geometry is also implemented (for neutral particles only).

Two variants of the second-order of accuracy adaptive weighted diamond difference scheme (AWDD scheme [3]) for spatial and angular variables are implemented: a) standard; b) with additional monotonization at strong material discontinuities. The AWDD scheme is also used for approximation of the continuous slowing-down term in solving the charged particle transport problems.

The P1 Synthetic Acceleration (P1SA) scheme for acceleration of inner iterations convergence, consistent with the WDD scheme, is implemented [4]. The consistent P1SA scheme for acceleration of fission upscattering iterations convergence in solving subcritical problems and thermal upscattering iterations convergence with the use of the estimated by Fourier analysis spectrum shape function for homogenized problem is also implemented. For solving the P1SA system for acceleration corrections the cyclic splitting-up method is used.

Parallelization of KATRIN code is performed via a 2-D spatial decomposition in $\mathrm{x}, \mathrm{y} / \mathrm{r}, \theta$ transverse section of the problem geometry, which retains the ability to invert the source iteration equation in a single sweep (the KBA algorithm). The solving of the P1 system for acceleration corrections is parallelized by performing the array of through-computation runs in parallel. Calculation of the scattering integral is parallelized in circular variable.

The code works both with symmetrical and asymmetrical angular meshes, a module that generates suitable quadrature meshes ( $\mathrm{ES}_{\mathrm{n}}$ type [5], Gauss-Chebychev and composite $\mathrm{S}_{\mathrm{n}}$ type (the last quadrature can be used in the case when it is necessary to give more nodes in the desirable angular direction)) is included in KATRIN. The number of discrete ordinates directions and the order of the $\mathrm{P}_{\mathrm{L}}$ approximation can vary in energy groups.

All calculations have been done in $\mathrm{P}_{3} \mathrm{~S}_{8}$ approximation using BGL1000_B7 [6] problemoriented nuclear data library (47 neutron groups) based on ENDF/B-VII.0. Cross-section library IRDF-2002 [7] and daily reactor power history were used for evaluation of neutron dosimeters activities.

Problem's geometry and materials are setting up by combinatorial geometry module. To perform the calculations detailed 3D model of VVER-1000/320 was created. This calculation model contains detailed description of reactor constructions including reactor core, internals, surveillance specimen's assemblies and ex-vessel cavity. Examples of the VVER-1000 combinatorial model with surveillance assemblies on the RPV are shown in Fig. 1. Combinatorial model is approximated by three-dimensional $R-\theta-Z$ grid using ConDat converter [8]. The size of $R-\theta-Z$ grid for VVER-1000 (Fig. 1) is about $310 \times 120 \times 520$. One of the most important features of code KATRIN is using volume fraction (VF) method that enables to keep the local mass and source balance during approximation of the actual reactor geometry and pin-by-pin neutron source.

\section{Testing of Russian 3D Code KATRIN for Fast Neutron Fluence Calculation on RPV of VVER-1000}

\subsection{Comparison with Surveillance Specimens Dosimetry Results}

The KATRIN calculations were tested with the latest VVER-1000 RPV dosimetry results. The most important experimental results which were used for validation are the activity measurements of the neutron dosimeters and fragments of specimens from surveillance assemblies irradiated during 5 years on the RPV inner surface. It is the first time the experimental data from surveillance capsules, irradiated close to the inner surface of RPV, have been obtained in Russia.

Locations of specimens and Neutron Dosimeters (ND) in surveillance containers are shown in Fig. 2. Measurements of neutron dosimeters activity and "retrospective" dosimetry measurements of ${ }^{54} \mathrm{Mn}$ activity accumulated in each irradiated specimen has been done in Kurchatov Institute. Comparison 


\section{$15^{\text {th }}$ ISRD}
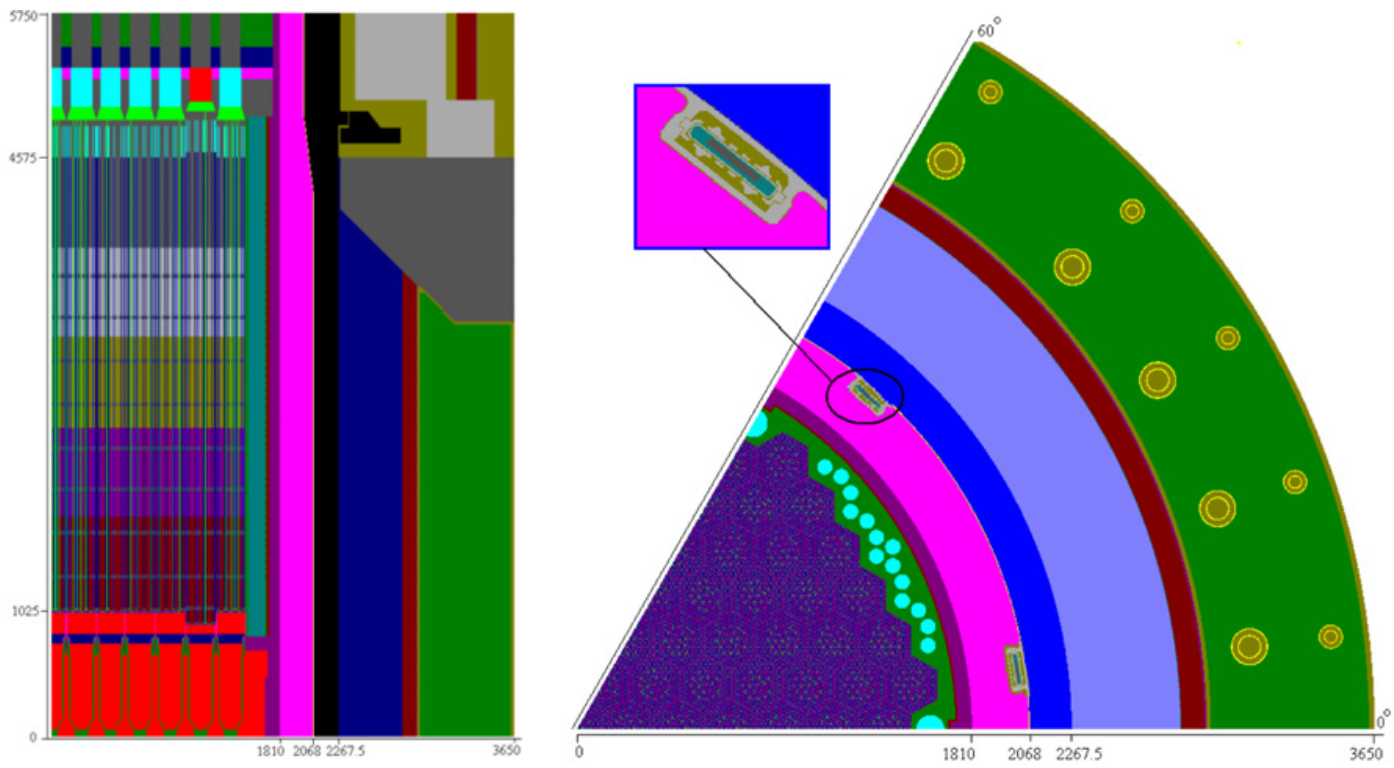

Figure 1. Fragments of VVER-1000 combinatorial model (dimensions in mm).
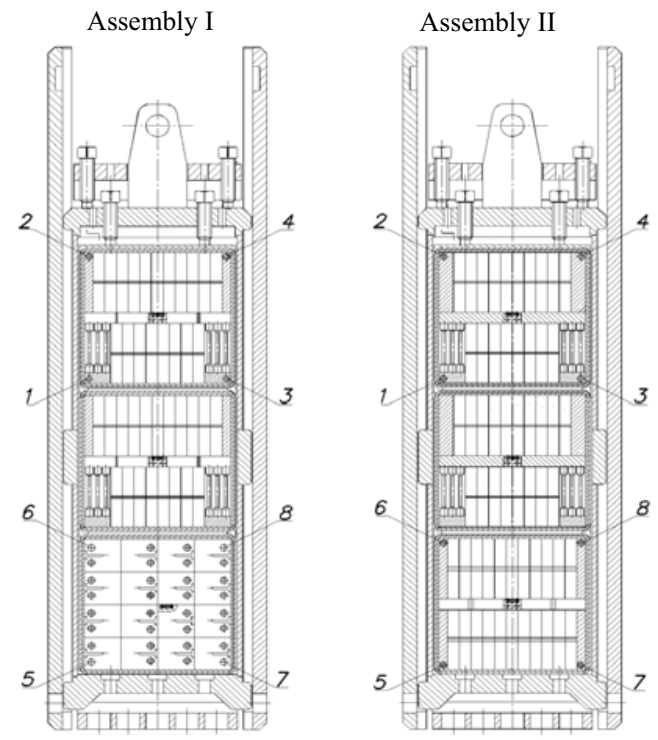

Figure 2. Disposition of neutron dosimeters (1-8) and surveillance specimens in containers.

of calculated and experimental (C/E) activities of ND is shown in Tables 1-3. Very good agreement between measurements and calculations is observed for all kinds of dosimeters. For most ND C/E value is within $2 \sigma$ error limits of dosimeters measurements. 
Table 1. Comparison of Calculated and Experimental activities of ${ }^{93 \mathrm{~m}} \mathrm{Nb}$ in niobium ND.

\begin{tabular}{|c|c|c|c|c|c|}
\hline \multirow{2}{*}{ Assembly } & \multirow{2}{*}{ Specimen } & \multicolumn{2}{|c|}{$A_{0}(93 \mathrm{~m} \mathrm{Nb}) \times 10^{-15} \mathrm{~Bq} / \mathrm{nucl}$} & \multicolumn{2}{c|}{$\mathrm{C} / \mathrm{E}$} \\
\cline { 3 - 6 } & & Experiment (E) & Calculation $(\mathrm{C})$ & By specimen & Average \\
\hline \multirow{3}{*}{$\mathrm{I}$} & 1 & 1.11 & 1.17 & 1.05 & \\
& 2 & 1.15 & 1.19 & 1.03 & \multirow{2}{*}{1.05} \\
& 3 & 1.08 & 1.16 & 1.07 & \\
\hline
\end{tabular}

Table 2. Comparison of Calculated and Experimental activities of ${ }^{60} \mathrm{Co}$ in copper ND.

\begin{tabular}{|c|c|c|c|c|c|}
\hline \multirow{2}{*}{ Assembly } & \multirow{2}{*}{ Specimen } & \multicolumn{2}{|c|}{$A_{0}\left({ }^{60} \mathrm{Co}\right), \times 10^{-17} \mathrm{~Bq} / \mathrm{nucl}$} & \multicolumn{2}{|c|}{$\mathrm{C} / \mathrm{E}$} \\
\hline & & Experiment (E) & Calculation $(\mathrm{C})$ & By specimen & Average \\
\hline \multirow{8}{*}{ I } & 1 & 1.65 & 1.65 & 1.00 & \multirow{4}{*}{1.01} \\
\hline & 2 & 1.70 & 1.66 & 0.98 & \\
\hline & 3 & 1.60 & 1.65 & 1.03 & \\
\hline & 4 & 1.62 & 1.66 & 1.03 & \\
\hline & 5 & 1.59 & \multirow{4}{*}{1.66} & - & \multirow{4}{*}{1.04} \\
\hline & 6 & 1.57 & & - & \\
\hline & 7 & 1.58 & & - & \\
\hline & 8 & 1.61 & & - & \\
\hline \multirow{8}{*}{ II } & 1 & 1.53 & 1.65 & 1.08 & \multirow{4}{*}{1.05} \\
\hline & 2 & 1.57 & 1.65 & 1.05 & \\
\hline & 3 & 1.57 & 1.65 & 1.05 & \\
\hline & 4 & 1.58 & 1.64 & 1.04 & \\
\hline & 5 & 1.57 & 1.63 & 1.03 & \multirow{4}{*}{1.05} \\
\hline & 6 & 1.55 & 1.62 & 1.05 & \\
\hline & 7 & 1.57 & 1.66 & 1.05 & \\
\hline & 8 & 1.54 & 1.66 & 1.07 & \\
\hline
\end{tabular}

Table 3. Comparison of Calculated and Experimental activities of ${ }^{54} \mathrm{Mn}$ in iron ND.

\begin{tabular}{|c|c|c|c|c|c|}
\hline \multirow{2}{*}{ Assembly } & \multirow{2}{*}{ Specimen } & \multicolumn{2}{|c|}{$A_{0}\left({ }^{54} \mathrm{Mn}\right), \times 10^{-15} \mathrm{~Bq} / \mathrm{nucl}$} & \multicolumn{2}{|l|}{$\mathrm{C} / \mathrm{E}$} \\
\hline & & Experiment (E) & Calculation (C) & By specimen & Average \\
\hline \multirow{8}{*}{ I } & 1 & 2.94 & 2.86 & 0.97 & \multirow{4}{*}{0.98} \\
\hline & 2 & 3.07 & 2.89 & 0.94 & \\
\hline & 3 & 2.86 & 2.92 & 1.02 & \\
\hline & 4 & 2.96 & 2.94 & 0.99 & \\
\hline & 5 & 2.85 & \multirow{4}{*}{2.92} & - & \multirow{4}{*}{1.01} \\
\hline & 6 & 2.89 & & - & \\
\hline & 7 & 2.85 & & _ & \\
\hline & 8 & 2.96 & & - & \\
\hline \multirow{8}{*}{ II } & 1 & 3.00 & 2.94 & 0.98 & \multirow{4}{*}{0.98} \\
\hline & 2 & 2.90 & 2.89 & 1.00 & \\
\hline & 3 & 2.97 & 2.92 & 0.98 & \\
\hline & 4 & 2.96 & 2.86 & 0.97 & \\
\hline & 5 & 3.03 & 2.88 & 0.95 & \multirow{4}{*}{0.97} \\
\hline & 6 & 2.93 & 2.82 & 0.96 & \\
\hline & 7 & 3.03 & 2.88 & 0.95 & \\
\hline & 8 & 2.93 & 2.94 & 1.00 & \\
\hline
\end{tabular}

\subsection{Comparison with Ex-Vessel Measurements}

Ex-vessel measurements for neutron fluence monitoring on RPV are recommended by Russian norms. The results of several ex-vessel measurements which were carried out on Russian VVER-1000 reactors were used for testing the KATRIN calculations.

Ex-vessel measurements provide 60-degree azimuth distributions of neutron dosimeters activity at the level of RPV welds and base metal. Axial distributions at the maximum region are optional. 

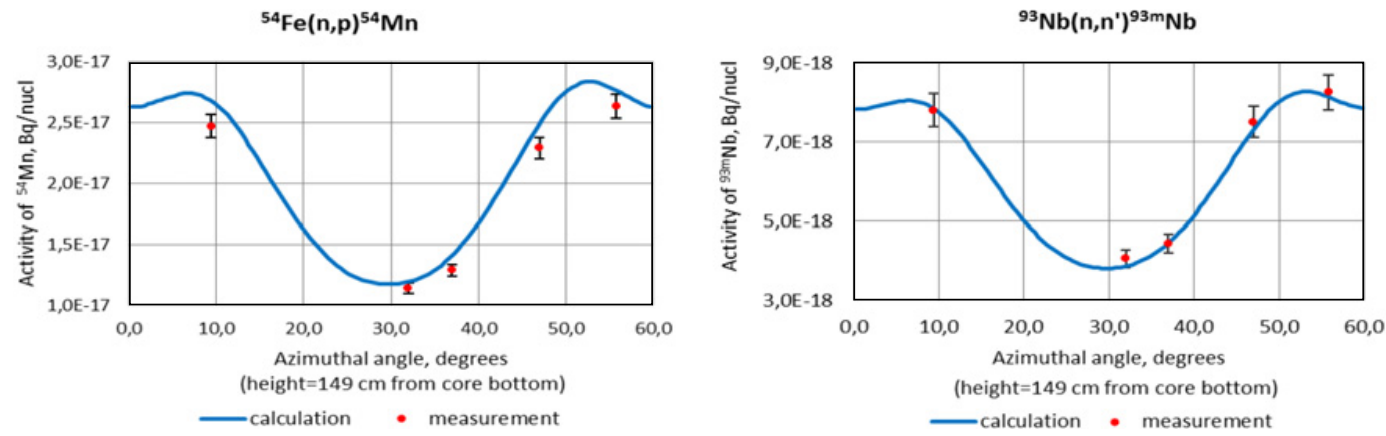

Figure 3. Azimuthal distribution of ${ }^{54} \mathrm{Mn}$ and ${ }^{93 \mathrm{~m}} \mathrm{Nb}$ activity at the axial maximum level (Balakovo NPP, unit 3).
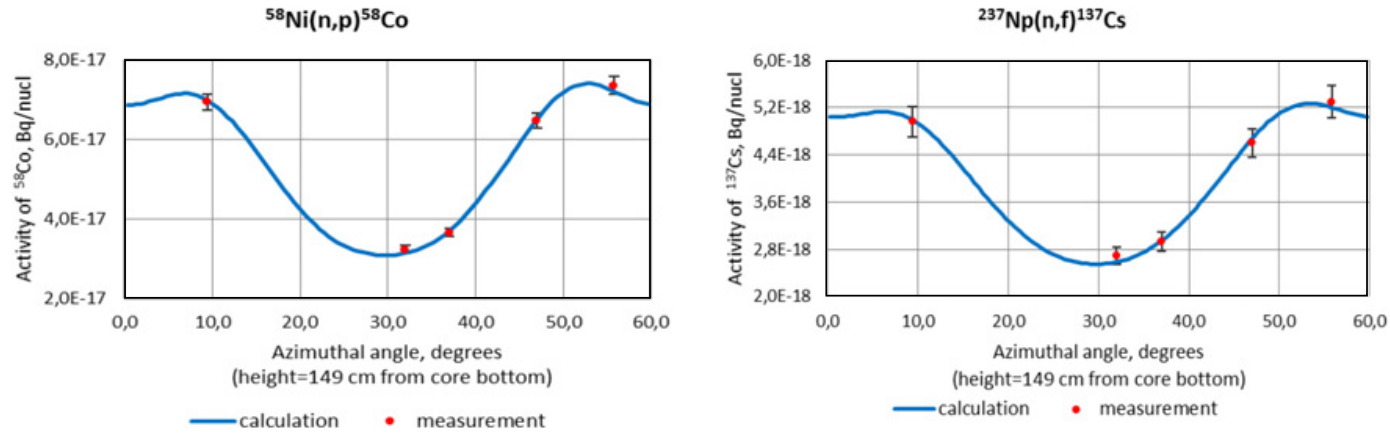

Figure 4. Azimuthal distribution of ${ }^{58} \mathrm{Co}$ and ${ }^{137} \mathrm{Cs}$ activity at the axial maximum level (Balakovo NPP, unit 3).
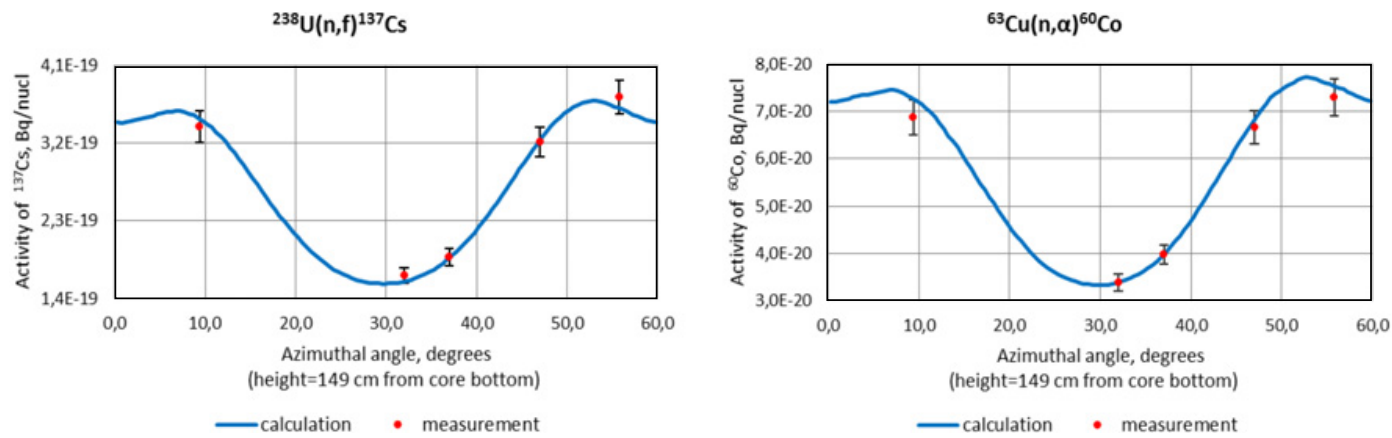

Figure 5. Azimuthal distribution of ${ }^{137} \mathrm{Cs}$ and ${ }^{60} \mathrm{Co}$ activity at the axial maximum level (Balakovo NPP, unit 3).

In the framework of international experiment carried out at unit 3 of Balakovo NPP [9] the following ND were irradiated on the outer surface of RPV: ${ }^{93} \mathrm{Nb}\left(n, n^{\prime}\right),{ }^{58} \mathrm{Ni}(n, p),{ }^{54} \mathrm{Fe}(n, p)$, ${ }^{63} \mathrm{Cu}(n, \alpha),{ }^{237} \mathrm{~Np}(n, f),{ }^{238} \mathrm{U}(n, f)$. Measurements have been carried out in several laboratories from different countries.

Comparison of calculated and experimental activities of different ND is shown in Figs. 3-5. Minimum, maximum and average $\mathrm{C} / \mathrm{E}$ ratios for different kind of neutron dosimeters are shown in Table 4. On average the agreement for all ND is sufficient and difference between calculations and experiment do not exceed $\pm 7 \%$. 
Table 4. C/E ratios for neutron dosimeters, Balakovo-3.

\begin{tabular}{|c|c|c|c|}
\hline \multirow{2}{*}{$\mathrm{ND}$} & \multicolumn{3}{|c|}{$\mathrm{C} / \mathrm{E}$} \\
\cline { 2 - 4 } & Min & Max & Average \\
\hline${ }^{93} \mathrm{Nb}\left(n, n^{\prime}\right)$ & 0.96 & 1.01 & 0.99 \\
\hline${ }^{58} \mathrm{Ni}(n, p)$ & 0.95 & 1.02 & 0.98 \\
\hline${ }^{54} \mathrm{Fe}(n, p)$ & 1.03 & 1.07 & 1.05 \\
\hline${ }^{63} \mathrm{Cu}(n, \alpha)$ & 0.95 & 1.05 & 1.02 \\
\hline${ }^{237} \mathrm{~Np}(n, f)$ & 0.96 & 1.01 & 0.98 \\
\hline${ }^{238} \mathrm{U}(n, f)$ & 0.96 & 1.03 & 0.99 \\
\hline
\end{tabular}
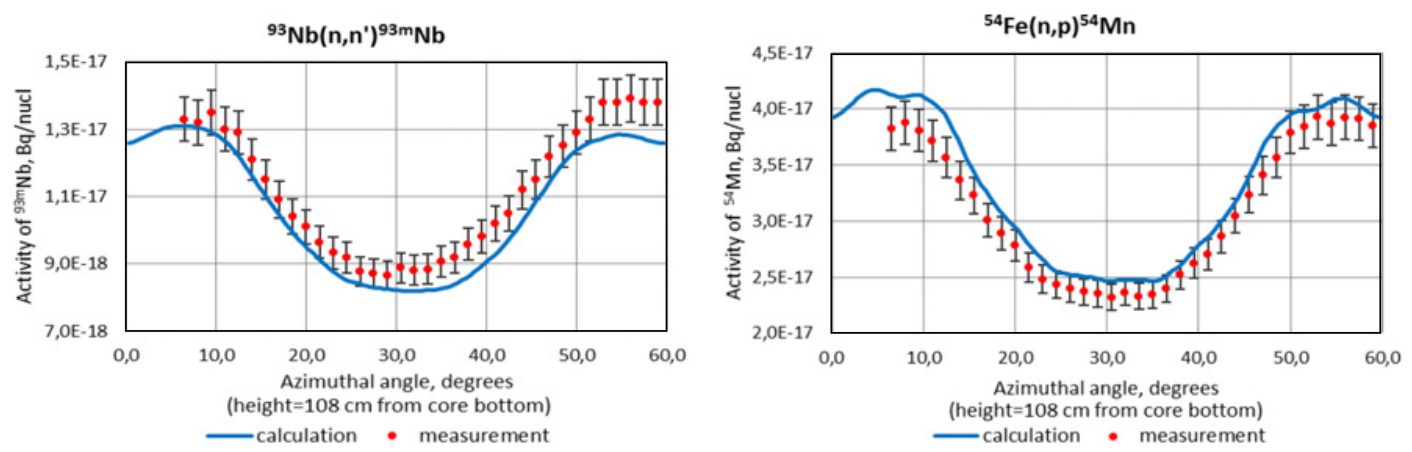

Figure 6. Azimuthal distribution of ${ }^{93 \mathrm{~m}} \mathrm{Nb}$ and ${ }^{54} \mathrm{Mn}$ activity at the axial maximum level (Balakovo NPP, unit 1 , cycle 18).
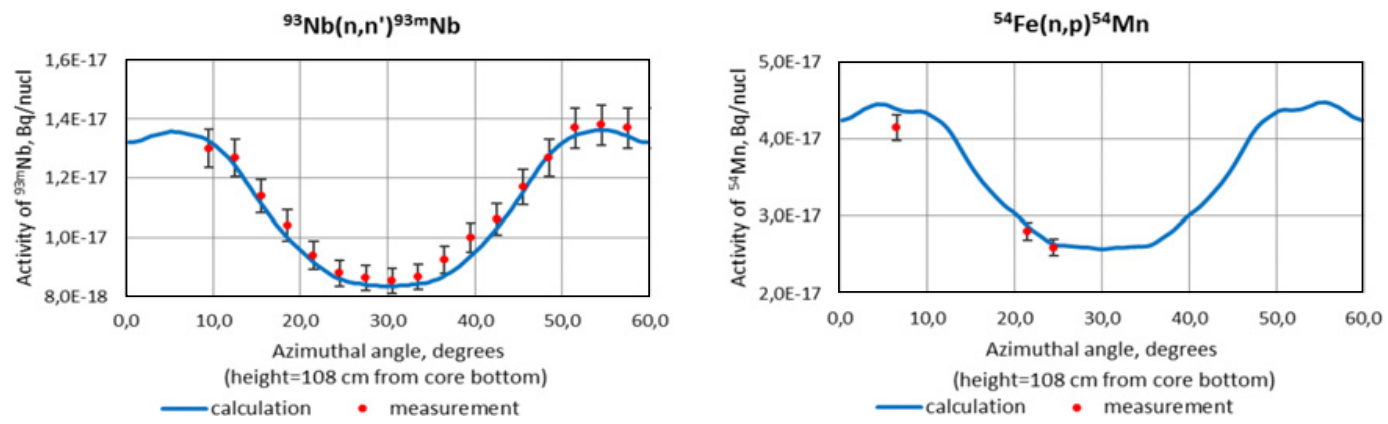

Figure 7. Azimuthal distribution of ${ }^{93 \mathrm{~m}} \mathrm{Nb}$ and ${ }^{54} \mathrm{Mn}$ activity at the axial maximum level (Balakovo NPP, unit 1 , cycle 19).

The similar technology is applied for regular ex-vessel monitoring of Russian VVER-1000 reactors. The regular ND sets consists of iron and niobium dosimeters.

In the paper comparison of KATRIN calculations with experimental data, obtained in such measurements in two cycles at Balakovo NPP has been done. ND activity were measured in Kurchatov Institute. Azimuthal distributions of calculated and measured activities of iron and niobium dosimeters are shown in Figs. 6, 7.

For iron dosimeters $\mathrm{C} / \mathrm{E}$ ratio is ranging from 0.99 to 1.06 (in average 1.06). For niobium dosimeters $\mathrm{C} / \mathrm{E}$ ratio is ranging from 0.92 to 1.01 (in average 0.94). Comparison shows good agreement between calculated and experimental ND activities and $\mathrm{C} / \mathrm{E}$ ratio is comparable to $2 \sigma$ error limits of dosimeters measurements. 


\section{$15^{\text {th }}$ ISRD}

\section{Conclusion}

The testing of Russian 3D code KATRIN for fast neutron fluence calculation on RPV is presented. KATRIN calculations have been compared with ex-vessel measurements at VVER-1000 and with the experimental results obtained for the first time in the surveillance assemblies irradiated close to inner surface of VVER-1000 RPV.

All the comparisons show very good agreement of calculation and experimental results. For most neutron dosimeters $\mathrm{C} / \mathrm{E}$ value is within $5-8 \%$. This value is comparable to $2 \sigma$ error limits of dosimeters measurements and is much less than theoretical uncertainty of fast neutron fluence calculation (about 20\%) [10].

Thus, the results obtained in this work confirmed the importance and necessity of regular ex-vessel activation measurements at VVER reactors and showed important role of experimental data comes from surveillance assemblies irradiated near vessel wall for approval of neutron fluence calculations at vessel. This conclusion is also compatible with modern Russian regulations, which deal with fast neutron fluence calculation approach.

It shall be noted that in spite of a great significance of the experimental results obtained at operating NPPs for more adequate validation of calculation of fast neutron flux on the reactor vessel using code KATRIN with regard of the applied approximations of the discrete ordinate method, it is reasonable to carry out comparison with the calculation results by Monte Carlo method. Some results of such comparison are presented in [11].

\section{References}

[1] A. M. Voloschenko, S. V. Gukov, A. A. Russkov, M. I. Gurevich, D. A. Shkarovsky, V. P. Kryuchkov, O. V. Sumaneev, A. A. Dubinin, "The CNCSN-2: One, Two- and Three-Dimensional Coupled Neutral and Charged Particle Discrete Ordinates Code System”, Proc. of International Conference on Advances in Mathematics, Computational Methods, and Reactor Physics, Saratoga Springs, USA, May 3-7, 2009

[2] A. M. Voloschenko and V. P. Kryuchkov, "KATRIN-2.0: Three-Dimensional Discrete Ordinates Neutron, Photon and Charged Particle Transport Code," User's Guide, Report of Keldysh Inst. of Appl. Math., Russian Ac. of Sci., No. 7-27-2004, Moscow, 2004

[3] R. E. Alcouffe. "An Adaptive Weigthed Diamond-Differencing Method for Three-Dimensional XYZ Geometry, TANS. 68A, 206 (1993)

[4] A. Voloschenko, "Consistent P1 Synthetic Acceleration Scheme for Transport Equation in 3D Geometries", Mathematics and Computation, Supercomputing, Reactor Physics and Nuclear and Biological Applications Palais des Papes, Avignon, France, September 12-15, 2005 on CD-ROM, American Nuclear Society, LaGrange Park, IL (2005)

[5] B. G. Carlson. "A method of characteristics and other improvements in solution methods for the transport equation", Nucl. Sci. Eng., 61, 408 (1976)

[6] A. Voloschenko, S. Zaritskiy, A. Egorov and V. Boyarinov. "Modernization of cross section library for VVER-1000 type reactors internals and pressure vessel dosimetry", Proc. of the 15-th International Symposium on Reactor Dosimetry, 2014, paper E133

[7] International Reactor Dosimetry File 2002 (IRDF 2002), Technical Reports Series No. 452, IAEA, Vienna, 2006

[8] M. I. Gurevich, A. A. Russkov, A. M. Voloschenko, "ConDat 1.0 - Code for Converting by the Tracing Algorithm the Combinatorial Geometry Presentation to the Bit-Mapped One, Users Guide," Preprint of Keldysh Inst. of Appl. Math., Russian Ac. of Sci., No. 12, 2007

[9] G.I. Borodkin and O.M. Kovalevich. Interlaboratory VVER-1000 Ex-vessel Experiment at Balakovo-3 NPP, Proc. of the 9th International Symposium on Reactor Dosimetry, Sep. 2-6, 
1996, Prague, Czech Republic. Hamid Ait Abderrahim et al. Eds., World Scientific Publishing Co. Pte. Ltd., 1998, pp. 431-438

[10] Calculated-Experimental estimation of Neutron Fluence on VVER-1000 Pressure Vessels for Lifetime Extension till 60 years, MT 1.2.3.06.0190-2013, Rosenergoatom (in Russian)

[11] M. I. Gurevich. A. A. Russkov. A. M. Voloshchenko. "Variance reduction in hybrid CADIS calculations of radiation shielding for VVER,” Atomic Energy, May 2011, 110, Issue 1, pp. 6-13 